\title{
Bone morphogenetic protein 9 stimulates callus formation in osteoporotic rats during fracture healing
}

\author{
XING WANG ${ }^{1}$, JUN HUANG $^{2}$, FAN HUANG $^{3}$, JIAN-CHUN ZONG $^{1}$, XI TANG $^{4}$, \\ YANG LIU $^{1}$, QIONG-FANG ZHANG ${ }^{5}$, YANG WANG ${ }^{1}$, LIANG CHEN ${ }^{1}$, \\ LIANG-JUN YIN $^{1}$, BAI-CHENG HE ${ }^{2}$ and ZHONG-LIANG DENG ${ }^{1}$
}

\author{
${ }^{1}$ Department of Orthopaedic Surgery, Second Affiliated Hospital of Chongqing Medical University; \\ ${ }^{2}$ Chongqing Key Laboratory of Biochemistry and Molecular Pharmacology, Chongqing Medical University, \\ Chongqing 400010, P.R. China; ${ }^{3}$ Center for Musculoskeletal Surgery, Charité-Universitätsmedizin, D-13353 Berlin, \\ Germany; ${ }^{4}$ Key Laboratory of Laboratory Medical Diagnostics, Ministry of Education, Chongqing Medical University, \\ Chongqing 400016; ${ }^{5}$ Key Laboratory of Molecular Biology for Infectious Diseases, Ministry of Education, \\ Second Affiliated Hospital of Chongqing Medical University, Chongqing 400010, P.R. China
}

Received November 18, 2015; Accepted November 22, 2016

DOI: $10.3892 / \mathrm{mmr} .2017 .6302$

\begin{abstract}
Fracture healing involves the coordinated actions of multiple cytokines. Bone morphogenetic protein 9 (BMP9) is an important factor in bone formation. The present study aimed to investigate the osteogenic potential of bone marrow stem cells (BMSCs) in response to adenoviral (Ad) BMP9, and the early fracture repair properties of AdBMP9 in surgically-created fractures in osteoporotic rats. Alkaline phosphatase (ALP) activity was assayed and matrix mineralization was examined by Alizarin Red S staining. mRNA and protein expression levels of BMP9, runt-related transcription factor 2 (RUNX2) and type 1 collagen (COL-1) were detected in vitro and in vivo. Femoral bone mineral density was assessed for osteoporosis in ovariectomized rats. An open femora fracture was subsequently created, and gelatin sponges containing AdBMP9 were implanted. The femora were harvested for radiographical, micro-computed tomography, biomechanical and histological analysis 4 weeks later. BMP9 successfully increased ALP activity and induced mineralized nodule formation in BMSCs. BMP9 in gelatin sponges demonstrated marked effects on microstructural parameters and the biomechanical strength of bone callus. In addition, it upregulated the expression levels of RUNX2 and COL-1. AdBMP9 in gelatin sponges significantly mediated callus formation, and increased bone mass and strength in osteoporotic rats with
\end{abstract}

Correspondence to: Dr Zhong-Liang Deng, Department of Orthopaedic Surgery, Second Affiliated Hospital of Chongqing Medical University, 74 Linjiang Road, Yuzhong, Chongqing 400010, P.R. China

E-mail: zhongliang_deng@126.com

Key words: bone marrow stem cells, bone morphogenetic protein 9, gelatin sponges, osteoporotic fracture, fracture healing femora fractures. The results of the present study suggested that BMP9 enhanced callus formation and maintained early mechanical stability during fracture healing in osteoporotic rats, implicating it as a potential novel therapeutic target for fracture healing.

\section{Introduction}

Osteoporotic fractures are a specific type of bone fracture resulting from mild impact, which may have serious consequences in osteoporotic patients. The estimated overall lifetime risk of any osteoporotic fracture is $13-22 \%$ in males and $40-50 \%$ in females (1); therefore, osteoporosis-associated fractures are a primary clinical and public health concern. Despite significant improvements in treatment strategies, a large number of patients suffer from delayed healing, fracture nonunion or other serious complications. Clinical investigations have increasingly focused on examining the ideal biological microenvironment for fracture healing and the prevention of nonunion. The extensive proliferation and differentiation potential of mesenchymal stem cells (MSCs) render them suitable for bone tissue engineering. However, the effectiveness of bone tissue engineering using MSCs relies on the predesigned scaffold and osteogenic factors used. Osteogenic factors, including bone morphogenetic proteins (BMPs), have been revealed as crucial scaffolding proteins in bone tissue engineering for osteogenic differentiation resulting in functional bone tissue (2).

BMP9 has been suggested to be the most osteo-inductive of various forms of recombinant BMPs, and may improve osteogenic differentiation of BMSCs in vitro and in vivo (3). Unlike BMP2 and BMP7, BMP9 may effectively induce orthotopic ossification that is not inhibited by BMP3, a known inhibitor of BMP2- and BMP7-mediated osteogenesis (4). Previous studies have indicated that BMP9 may induce osteogenic differentiation of primary osteoblasts, pre-osteoblasts and other directed-differentiated stem cells $(5,6)$, suggesting a potential 
role for BMP9 in promoting the shift of directed-differentiated stem cells towards osteoblastic differentiation. Additionally, previous in vivo studies have indicated that adenovirus (Ad)BMP9 or AdBMP9-transfected human MSCs induce spinal fusion in rodents $(7,8)$. Furthermore, injection of AdBMP9-transduced osteoblast progenitors or viral vectors into the quadriceps of athymic mice effectively induced orthotopic ossification (4). Shui et al (9) demonstrated that subcutaneous implantation of BMP9-engineered cells with a type 1 collagen (COL-1) sponge or hydroxyapatite-tricalcium phosphate scaffold resulted in robust and mature cancellous bone masses, compared with minimal bone formation using demineralized bone matrix. However, few studies have focused on the effect of BMP9 on fracture healing in osteoporotic rats. The present study hypothesized that BMP9 may mediate callus formation and fracture healing in osteoporotic rats. The osteoconductive activities and bone regeneration potential of a gelatin sponge containing AdBMP9 were evaluated in rats with osteoporotic fractures.

\section{Materials and methods}

Isolation and culture of bone marrow stromal cells (BMSCs). All animal procedures were approved by the Animal Research Ethics Committee of Chongqing Medical University (Chongqing, China). Female Sprague Dawley rats ( $\mathrm{n}=50$; age, 3 months; weight, $276.6 \pm 18.9 \mathrm{~g}$ ) were obtained from the Animals Research Center of Chongqing Medical University (ARCCMU) (Chongqing, China). The animals were maintained at a constant temperature $\left(22 \pm 2^{\circ} \mathrm{C}\right)$ and humidity $(50 \%)$ under a 12-h light/dark cycle with free access to water and food. Primary BMSCs were harvested from the femora of rats as previously described (10). Animals were anesthetized with an intraperitoneal injection of sodium pentobarbital (200 mg/kg body weight, Sigma-Aldrich Merck Millipore, Darmstadt, Germany) and sacrificed by cervical dislocation. Cells at passage four were plated at $1 \times 10^{5} /$ well in 24-well plates and cultured in the Dulbecco's modified Eagle's medium (DMEM) or basal medium Eagle supplemented with $10 \%$ fetal bovine serum (FBS) and 5\% penicillin-streptomycin (all purchased from Gibco; Thermo Fisher Scientific, Inc., Waltham, MA, USA).

Recombinant adenoviruses expressing BMP-9. The AdEasy Adenoviral Vector system, was provided by Dr. Tongchuan He, was used to generate recombinant adenoviruses expressing BMP9 as previously described (11). The coding region of human BMP9 was amplified and cloned into an adenoviral shuttle vector to generate recombinant adenoviruses in Human Embryonic Kidney 293 cells [HEK293; American Type Culture Collection (ATCC), Manassas, VA, USA]. Adenoviruses were purified by cesium chloride gradient centrifugation at $176,000 \mathrm{x} g$ for $20 \mathrm{~h}$ at $10^{\circ} \mathrm{C}$. The virus titer was assessed by measuring absorbance at a wavelength of $260 \mathrm{~nm}$ prior to use. The resulting adenoviruses were designated as AdBMP9. Analogous adenoviruses expressing only monomeric green fluorescent protein (GFP; AdGFP) served as a control.

Preparation of conditioned medium (CM). The CM was prepared as previously described (12). HCT116 human colon carcinoma cells (ATCC) susceptible to adenovirus infection were used to secrete overexpressed proteins into the medium $(13,14)$. Cells were infected with an optimal titer of AdGFP or AdBMP9 for $24 \mathrm{~h}$. The culture medium was subsequently replaced with serum-free DMEM; the CM of AdGFP or AdBMP9 was collected $48 \mathrm{~h}$ after infection and used immediately.

$R N A$ extraction and reverse transcription-polymerase chain reaction $(R T-P C R)$ analysis. BMSCs were seeded into a 24-well plate and cultured for $24 \mathrm{~h}$. Cells were subsequently treated with AdGFP or AdBMP9 for $24 \mathrm{~h}$ in the presence of $8 \mu \mathrm{g} / \mathrm{ml}$ polybrene (Sigma-Aldrich; Merck Millipore) and observed via microscopy (Nikon Corporation, Tokyo, Japan). Following treatment with DMEM, AdGFP or AdBMP9 for 2 or 7 days, total RNA was isolated using TRIzol ${ }^{\circledR}$ reagent (Invitrogen; Thermo Fisher Scientific, Inc.) for expression analysis of BMP9 forward 5'-CGCAGCCTTAAC CTCAGC-3', reverse 5'-GTTGGAGGCAGGCGTAGA-3', runt-related transcription factor 2 (RUNX2) forward 5'-GCC AATCCCTAAGTGTGGCT-3', reverse 5'-AACAGAGAG CGAGGGGGTAT-3' and COL-1 forward 5'-CAGTCGCTT CACCTACAGCA-3', reverse 5'-GGTGGAGGGAGTTTA CACGA-3'. The cDNA templates were synthesized by RT reaction with hexamer and Superscript II RT (Invitrogen; Thermo Fisher Scientific, Inc.). The first strand cDNA products were further diluted 5- to 10-fold and used as PCR templates. Semi-quantitative PCR was carried out as described previously (15).

Protein extraction and western blot analysis. Protein extraction and western blotting was performed as previously described (16). To extract total proteins, cells treated with DMEM, AdGFP or AdBMP9 for 2 or 7 days were washed with cold PBS $\left(4^{\circ} \mathrm{C}\right)$ and lysed in $300 \mu$ RIPA lysis buffer (Sigma-Aldrich; Merck Millipore). Total proteins (30 $\mu \mathrm{g})$ were separated by $10 \%$ SDS-PAGE, transferred to polyvinylidene difluoride membranes, blocked in $10 \%$ skimmed milk, and probed at $4^{\circ} \mathrm{C}$ for $24 \mathrm{~h}$ with the following primary antibodies: Mouse anti-BMP9 (cat. no. sc-514211; 1:1,000; Santa Cruz Biotechnology, Inc., Dallas, TX, USA), rabbit anti-RUNX2 (cat. no. sc-10758; 1:1,000; Santa Cruz Biotechnology, Inc.), mouse anti-COL-1 (cat. no. ab6308; 1:1,000; Abcam, Hong Kong, China) and anti-GAPDH (cat. no. sc-32233; 1:1,000; Santa Cruz Biotechnology, Inc.), which served as the control. The membranes were subsequently incubated with the appropriate horseradish peroxidase-conjugated secondary antibodies, including goat anti-rabbit IgG (cat. no. sc-2004; 1:3,000; Santa Cruz Biotechnology, Inc.) or goat anti-mouse IgG (cat. no. sc-2005; 1:3,000; Santa Cruz Biotechnology, Inc.), at $25^{\circ} \mathrm{C}$ for $1 \mathrm{~h}$. Images of target bands were developed using the Thermo Scientific ${ }^{\mathrm{TM}}$ SuperSignal West Pico Chemiluminescent Substrate (Thermo Fisher Scientific, Inc.). The Quantity One version 4.62 software (Bio-Rad Laboratories, Inc., Hercules, CA, USA) was applied to assess the gray value and the rate of grey value between target band and control band was regarded as the relative expression level of target protein.

Alkaline phosphatase (ALP) activity assay. ALP activity was assessed using a modified Great EscAPe ${ }^{\mathrm{TM}}$ SEAP 
Chemiluminescence assay (Clontech Laboratories, Inc., Mountainview, CA, USA) and/or histochemical staining as described previously (17). BMSCs were treated with DMEM, CM-GFP or CM-BMP9 for 7, 9 and 11 days. Each Chemiluminescence assay was performed in triplicate and ALP activity was normalized against total cellular protein levels.

Matrix mineralization assay. Matrix mineralization was detected by Alizarin Red S (Sigma-Aldrich; Merck Millipore) staining as described previously (15). BMSCs were treated with DMEM, CM-GFP or CM-BMP9 for 28 days. The staining of calcium mineral deposits was recorded under bright-field microscopy. To quantify the matrix mineralization, Alizarin Red S was extracted with $1 \mathrm{ml} /$ well 100 mmol/l cetylpyridinium chloride (Sigma-Aldrich; Merck Millipore) and absorbance was measured at a wavelength of $570 \mathrm{~nm}$.

Osteoporotic fracture models. Previous studies have indicated that body weight (BW) and/or body mass index have an effect on bone mineral density and osteoporotic fractures (18-22). To reduce potential experimental errors caused by body weight variation in the present study, rats were randomly divided into sham $(n=10)$ and ovariectomized (OVX; $n=40)$ groups, and anesthetized with an intraperitoneal injection of sodium pentobarbital (200 mg/kg body weight) prior to surgery. Rats in the OVX group were bilaterally ovariectomized and rats in the sham group underwent a sham operation. After 3 months without any treatment for osteoporosis induction, 10 randomly selected animals in the OVX group and all 10 animals in the sham group were euthanized as previously described (23). The BW and uterus weight (UW) of rats were recorded, and femoral bone mineral density (BMD) was measured using dual energy X-ray absorptiometry (DXA). Following this, the femora were fixed in $4 \%$ paraformaldehyde at $4^{\circ} \mathrm{C}$ for $48 \mathrm{~h}$ and stored in $70 \%$ ethanol. DXA was performed using the Dual-Energy X-ray Absorption (XR-46; Norland Corp., Fort Atkinson, WI, USA) with Small Animal software version 2.5.0 (Norland Corporation, Fort Atkinson, WI, USA).

The remaining $30 \mathrm{OVX}$ rats were randomized into two groups: AdGFP and AdBMP9 ( $\mathrm{n}=15$ per group). Rats were anaesthetized as previously described. To create an open fracture, the left femur was prepared for surgery under standard sterile conditions. The patella was deflected laterally and a hole was drilled through the intercondylar notch of the femur using a mini electric drill (drill bit, 1.0-mm diameter). The periosteum of the femur was circumferentially incised and elevated, and a transverse osteotomy was made at the distal tuberositas deltoidea of the left femur with a mini electric saw. A 1-mm diameter Kirschner wire (length, $3 \mathrm{~cm}$; bend, $90^{\circ}$; handle, $3 \mathrm{~mm}$ ) was subsequently buried beneath the muscle and inserted through the hole across the fracture ends. Gelatin sponges (5x10-mm strips, Xiang En Jiangxi Medical Technology Development Co., Nanchang, China) soaked with $300 \mu \mathrm{l}$ AdGFP or AdBMP9 (2.2x10 12 viral particles/ml) were wrapped around the fracture site circumferentially. The muscle and skin incisions were sutured with 4-0 nylon suture (Johnson \& Johnson Medical Ltd., Wokingham, UK). Following surgery, plain X-rays were performed to confirm a proper fracture pattern. All rats were subsequently sacrificed after 4 weeks.

Radiographic analysis. After 4 weeks, the rats were anesthetized as previously described and radiographed in lateral planes for X-ray analysis using the RADspeed General Radiographic system (50 kv; 200 mv; 32 msev; Shimadzu Corporation, Kyoto, Japan) for high-precision focus detection.

Micro-computed tomography (CT) analysis. For analysis of fracture healing, animals ( $\mathrm{n}=5$ per group) were scanned using an eXplore Locus SP Pre-Clinical Specimen Micro-CT (GE Healthcare Life Sciences, Chalfont, UK) with an isotropic voxel resolution of $14 \mu \mathrm{m}$ (24). Rats were fastened onto a foam board and scanned perpendicular to the long bone axis with a tube voltage of $80 \mathrm{kV}$ and current of $80 \mathrm{~mA}$. A constrained 3D Gaussian filter was used to partially improve noise volumes. The fracture line of each specimen was measured as the central position for the region of interest, with the upper and lower $5 \mathrm{~mm}$ as the two margins. Cortical bone, callus bone and non-bony tissue were segmented by thresholding algorithm, following the manufacturer's protocol. Bone analysis was conducted using MicroView software version 2.1.2 (GE Healthcare Life Sciences). The output density data (Hounsfield units) was converted to mineral content (mg/cc) using the density data from the phantoms. For femoral analysis, bone volume (BV), BV/total volume (TV), bone mineral content (BMC) and BMD were calculated.

Biomechanical assessment. The fractured femora of each rat was harvested with saline-moistened gauze and stored at $-80^{\circ} \mathrm{C}$. Prior to biomechanical assessment, the intramedullary wires were removed. The femora ( $n=5$ per group) were subsequently subjected to a three-point bending assessment using the Instron 4302 Universal Testing system (Instron, Norwood, MA, USA). The fracture samples were prepared to remove the soft tissue. To maintain consistency between samples, all mechanical tests were conducted by one individual. The femora were placed in the material testing machine on two supports separated by a distance of $1.5 \mathrm{~mm}$, and the testing area was defined as the central part of the callus. A compression load was applied at a rate of $2 \mathrm{~mm} / \mathrm{min}$ until breakage. The data of ultimate load at failure $(\mathrm{N})$ and stiffness $(\mathrm{N} / \mathrm{mm})$ were monitored by a connected computer.

Histological analysis. The specimens evaluated by micro-CT analysis were decalcified with $10 \%$ EDTA disodium salt for 4 weeks. The tissues were subsequently embedded in paraffin and $5-\mu \mathrm{m}$ thick consecutive sections were cut using a hand-operated microtome (Leica Microsystems $\mathrm{GmbH}$, Wetzlar, Germany). The sections were stained with hematoxylin and eosin (H\&E) or Masson's trichrome, and the images were quantified as described in our previous study (15).

Callus protein extraction and western blot analysis. A total of 18 female Sprague Dawley rats (age, 6-months; weight, $321.3 \pm 7.7 \mathrm{~g}$ ) were obtained from ARCCMU and housed in the aforementioned conditions. Rats were randomly divided into AdGFP $(n=9)$ and AdBMP9 $(n=9)$ groups and the open fracture was created. All the fractured animals were treated with 
AdGFP or AdBMP9. At each time-point (days 7, 14 and 28), three animals per group were sacrificed, and the callus at the fracture site was collected and stored in liquid nitrogen. The frozen tissue samples harvested from the callus were homogenized and proteins were extracted for western blot analysis of BMP9, RUNX2 and COL-1 expression levels as described above.

Statistical analysis. Statistical analysis was performed using SPSS software version 20.0 (IBM SPSS, Armonk, NY, USA). All results are expressed as the mean \pm standard error. Differences between two groups were assessed using the unpaired Student's t-test. Comparisons of multiple groups were performed using one-way analysis of variance, followed by Turkey's post hoc test. $\mathrm{P}<0.05$ was considered to indicate a statistically significant difference.

\section{Results}

AdBMP9 promotes osteoblastic differentiation of BMSCs in vitro. BMSCs infected with AdBMP9 or AdGFP for $24 \mathrm{~h}$ were observed via microscopy (Fig. 1A). BMP9 mRNA and protein expression levels were significantly enhanced in the AdBMP9 group compared with the AdGFP and control groups $(\mathrm{P}<0.001$; Fig. 1B). To investigate the BMP9-induced osteogenic differentiation of BMSCs, ALP activity was measured following treatment of BMSCs with CM-BMP9 or CM-GFP for 7, 9 and 11 days (Fig. 1C). The activity of ALP was significantly enhanced in the CM-BMP9-treated group, peaking at day $9(\mathrm{P}<0.001)$. In addition, cells were stained with Alizarin Red $\mathrm{S}$ at day 28 (Fig. 1D), and the matrix mineralization was markedly induced by BMP9 as demonstrated by significantly greater Alizarin Red S staining in the AdBMP9, compared with the AdGFP and control, groups $(\mathrm{P}<0.001)$. Furthermore, whether BMP9 modulates the expression levels of the two osteogenesis-regulating proteins, RUNX2 and COL-1, was investigated. It was revealed that stable overexpression of BMP9 in BMSCs effectively upregulated RUNX2 (day 2) and COL-1 (day 7) mRNA and protein expression levels compared with the AdGFP and control groups $(\mathrm{P}<0.001$; Fig. 1B).

Animal health and DXA analysis. At the end of the experiment, three animals were excluded. In the process of creating the open fracture, one rat in the AdBMP9 group died from asphyxia. During fracture healing, one rat in the AdBMP9 group was excluded due to osteomyelitis and one in the AdGFP group due to distal migration of the nail. This left 14 rats in the AdGFP group and 13 rats in the AdBMP9 group.

Ovariectomy resulted in significant alterations in BW, UW and femoral BMD after 3 months. BW and UW differed significantly between the sham and OVX groups. In the OVX group, BW was significantly increased compared with the sham group $(366.5 \pm 7.32$ vs. $326.8 \pm 6.35 \mathrm{~g}$; $\mathrm{P}=0.001$; Fig. $2 \mathrm{~A})$. However, the UW of rats in the sham group was significantly increased compared with the OVX group $(1,002 \pm 59.02$ vs. 331.7 $\pm 28.89 \mathrm{mg}$; $\mathrm{P}<0.001$; Fig. 2B). The femoral BMD value in the OVX group was significantly reduced compared with the sham group $\left(0.2341 \pm 0.0020\right.$ vs. $0.2608 \pm 0.0019 \mathrm{mg} / \mathrm{cm}^{2} ; \mathrm{n}=10$; $\mathrm{P}<0.001$; Fig. 2C). These results demonstrated the successful establishment of osteoporosis in OVX rats.
Radiography, micro-CT and mechanical analysis of fracture callus. Following dissection of soft tissue from the bone, visual inspection revealed that AdBMP9-treated bone calluses appeared stronger and larger compared with those from the AdGFP-treated group. X-rays of the fractured femora demonstrated thicker calluses, suggesting an increased bone mass in AdBMP9-treated animals after 4 weeks (Fig. 3Aa). Ectopic bone formation was observed in one rat from the AdBMP9 group (data not shown). The $3 \mathrm{D}$ reconstructions of fractured femurs revealed that fracture gaps contained numerous calluses in the AdBMP9 group; however, these were almost undetectable in the AdGFP group. The transverse, sagittal and coronal micro-CT slices through the center of the fracture plate revealed that the newly-formed bone calluses were larger and denser in AdBMP9-treated animals compared with AdGFP-treated controls (Fig. 3Ab). The quantitative results of BV, BV/TV, BMC and BMD are presented in Fig. 3B. AdBMP9 treatment significantly increased BV by $23.9 \%$ and BV/TV by $25.0 \%$ $(\mathrm{P}<0.001)$, compared with the AdGFP group. Compared with the AdGFP group, BMC and BMD in the AdBMP9 group increased by 34.5 and $36.1 \%$ of the AdGFP group, respectively $(\mathrm{P}<0.001)$.

Results of biomechanical tests of fractured femora are presented as stiffness $(\mathrm{N} / \mathrm{mm})$ and ultimate load $(\mathrm{N}$; Fig. 3Be and f). In the AdBMP9 group, a marked $197.8 \%$ increase in mechanical stiffness was observed compared with the AdGFP group $(\mathrm{P}<0.001)$. Additionally, BMP9 significantly increased the ultimate load of the callus by $357.1 \%$ compared with the AdGFP group $(\mathrm{P}<0.001)$.

Histological analysis. In longitudinal sections stained with H\&E and Masson's trichrome, periosteal activation was present resulting in bony callus formation at the fracture ends, and the soft callus was present consisting of fibrous tissue with chondrogenic differentiation in and around the fracture gap (Fig. 4A and B). The AdBMP9 group demonstrated a cartilaginous tissue bridge (Fig. 4Ad and Bd); however, the AdGFP group revealed fibrous contact or no bridging (Fig. 4Ac and $\mathrm{Bc}$ ). Consistent with the BMD of the fractured femur, as determined by micro-CT, the decalcified callus appeared more compact in the AdBMP9 treated group compared with the AdGFP group (Fig. 4Ad and $\mathrm{f}$ and Bd and f). The AdBMP9 group demonstrated bone ossification and the presence of chondrocytes, indicating previous cellular differentiation (Fig. 4Ad and Bd). In the AdBMP9 group, the trabecular bone was more mature compared with the AdGFP group, as indicated by areas stained red, suggesting that BMP9 accelerated callus remodeling (Fig. 4Bb and f). Quantitative analysis of the trabecular area percentage of the total area revealed an increase in the AdBMP9 group compared with the AdGFP group $(\mathrm{P}=0.001$; Fig. 4C).

AdBMP9 induces callus formation by promoting osteoblastic differentiation in vivo. Protein expression levels of BMP9 were detected in the fracture callus at days 7, 14 and 28 post-surgery. BMP9 expression levels in the AdBMP9 group were significantly increased compared with the AdGFP group ( $\mathrm{P}=0.001)$. Relative BMP9 expression levels in bone callus from the AdBMP9 group peaked 
A

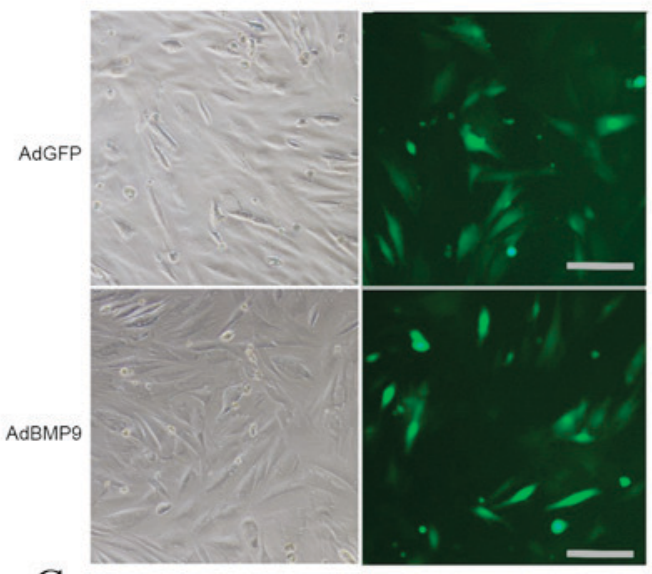

$\mathrm{C}$

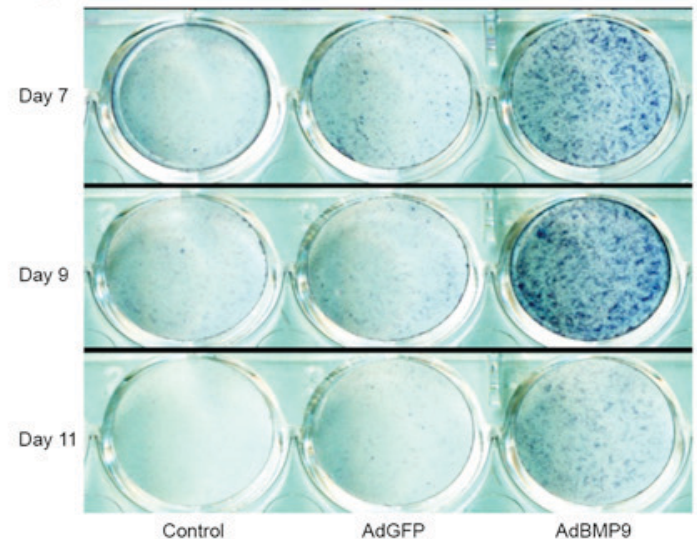

$\mathrm{B}$
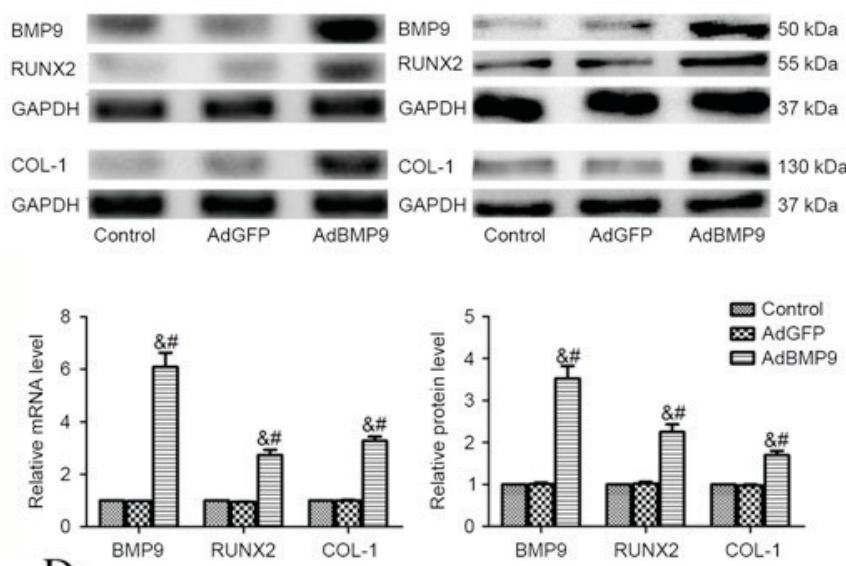

$\mathrm{D}$
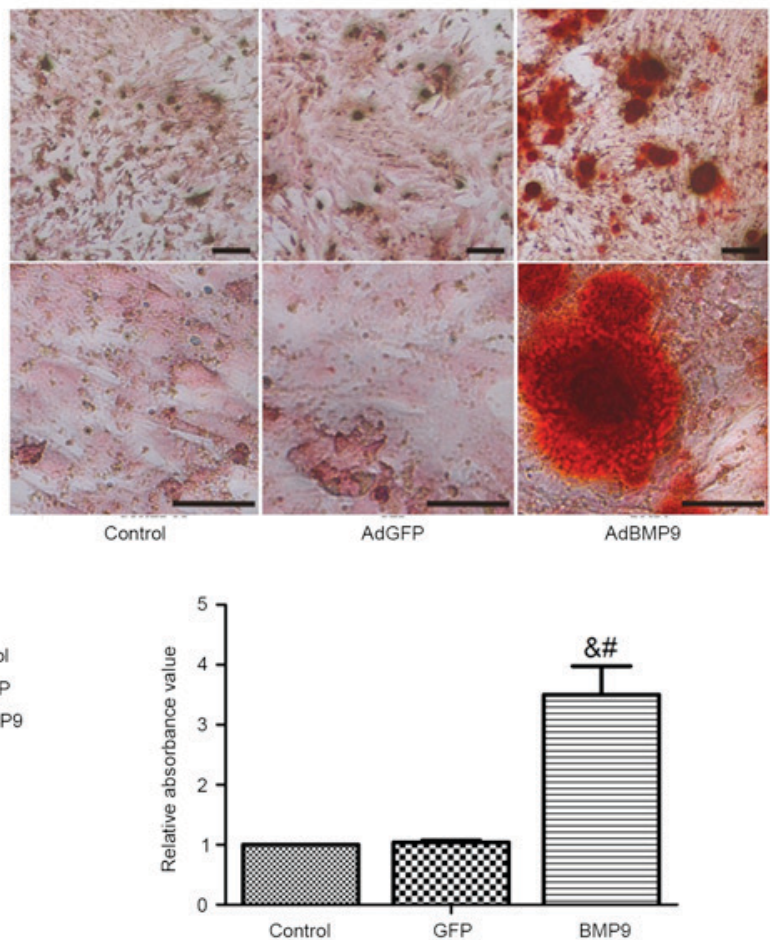

Figure. 1 AdBMP9 induces osteoblastic differentiation in vitro. (A) Representative images of the morphology of BMSCs transfected with AdBMP9 and AdGFP, and GFP signal, as observed via fluorescence microscopy 3 days following transfection (magnification, x100; scale bar=100 $\mu \mathrm{m}$ ). (B) mRNA and protein expression level analysis of BMP9, RUNX2 (day 2) and COL-1 (day 7) in BMSCs transfected with AdGFP or AdBMP9, or without transfection as a control. (C) ALP activity assay following BMSC treatment with CM-BMP9 or CM-GFP for 7,9 and 11 days. (D) Matrix mineralization assay following CM-BMP9 or CM-GFP treatment for 28 days, visualized using Alizarin Red S staining. Magnification, x40 (top row) or x200 (bottom row); scale bar=200 (top row) or $20 \mu \mathrm{m}$ (bottom row). Each assay was performed in triplicate. Data are presented as the mean \pm standard error. ${ }^{\text {\& }} \mathrm{P}<0.001 \mathrm{vs}$. control group, ${ }^{\prime \prime} \mathrm{P}<0.001$ vs. AdGFP group. Ad, adenoviral; BMSCs, bone marrow stem cells; GFP, green fluorescent protein; BMP9, bone morphogenic protein 9; CM, conditioned medium; ALP, alkaline phosphatase; RUNX2, runt-related transcription factor 2; COL-1, type 1 collagen.

at day 14 post-surgery (Fig. 5A). Although BMP9 expression levels in the AdBMP9 group declined at day 28, they remained significantly greater compared with the AdGFP group $(\mathrm{P}=0.017)$.

Protein expression levels of RUNX2 and COL-1 were examined by western blotting at days 7 and 14 post-surgery. RUNX2 expression levels in the AdBMP9 group were significantly increased at the two time points compared with the AdGFP group ( $\mathrm{P}=0.009$ and $\mathrm{P}=0.047$, respectively), whereas protein expression levels of COL-1 were only markedly enhanced at day 14 ( $\mathrm{P}=0.009$; Fig. 5B).

\section{Discussion}

In the present study, radiographic, biomechanical and histomorphometric analysis indicated that BMP9 in gelatin sponges may mediate callus formation in osteoporotic rats. A total of four weeks after implantation, BMP9 treatment significantly increased the formation and microstructure of bone callus, and improved healing, in rats with osteoporotic fractures, compared with the AdGFP control group. Furthermore, BMP9 in gelatin sponges successfully enhanced osteogenesis and upregulated the expression levels of RUNX2 and COL-1. 
A

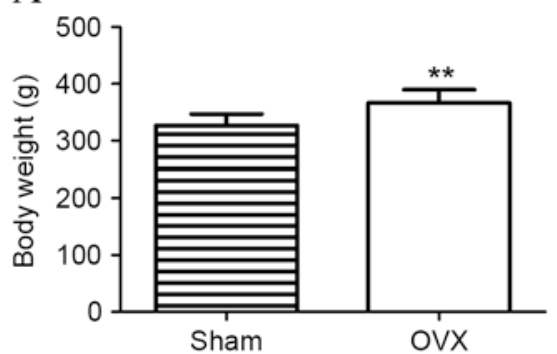

B

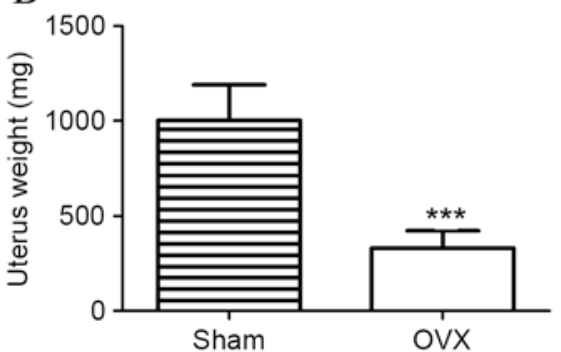

C

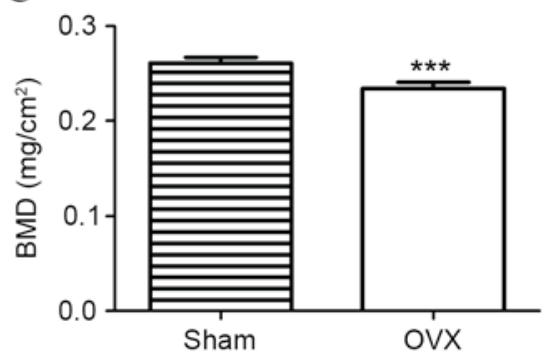

Figure 2. Rat osteoporotic models induced by ovariectomy. After 3 months, (A) Body and (B) uterus weights, and (C) BMD were quantified and analyzed. Data are presented as the mean \pm standard error $(\mathrm{n}=10) .{ }^{* *} \mathrm{P}<0.01$ and ${ }^{* * *} \mathrm{P}<0.001$ vs. sham group. BMD, bone mineral density.

A

a

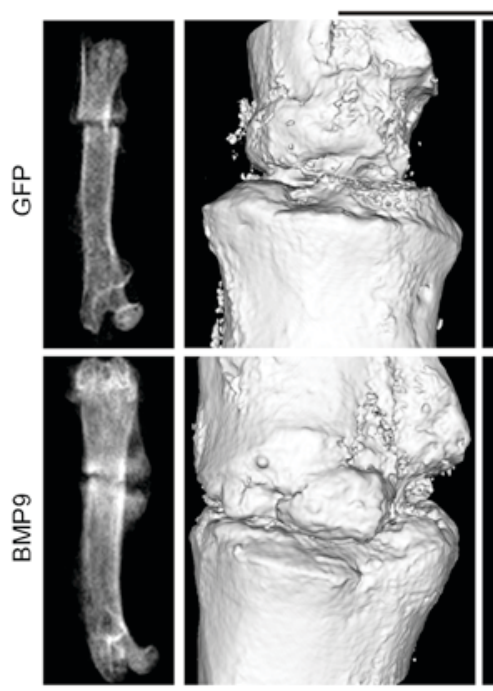

$\mathrm{b}$

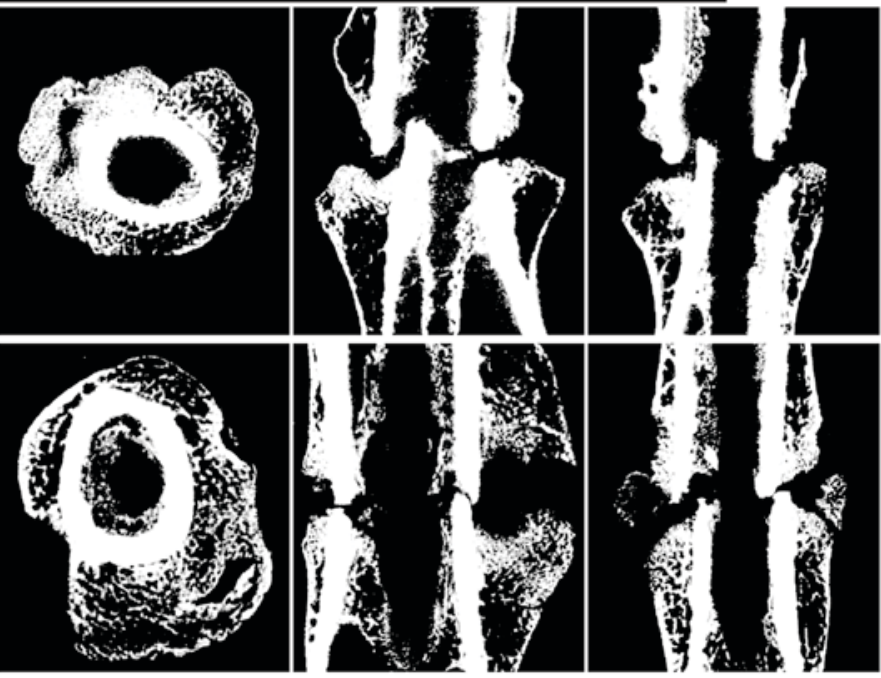

B
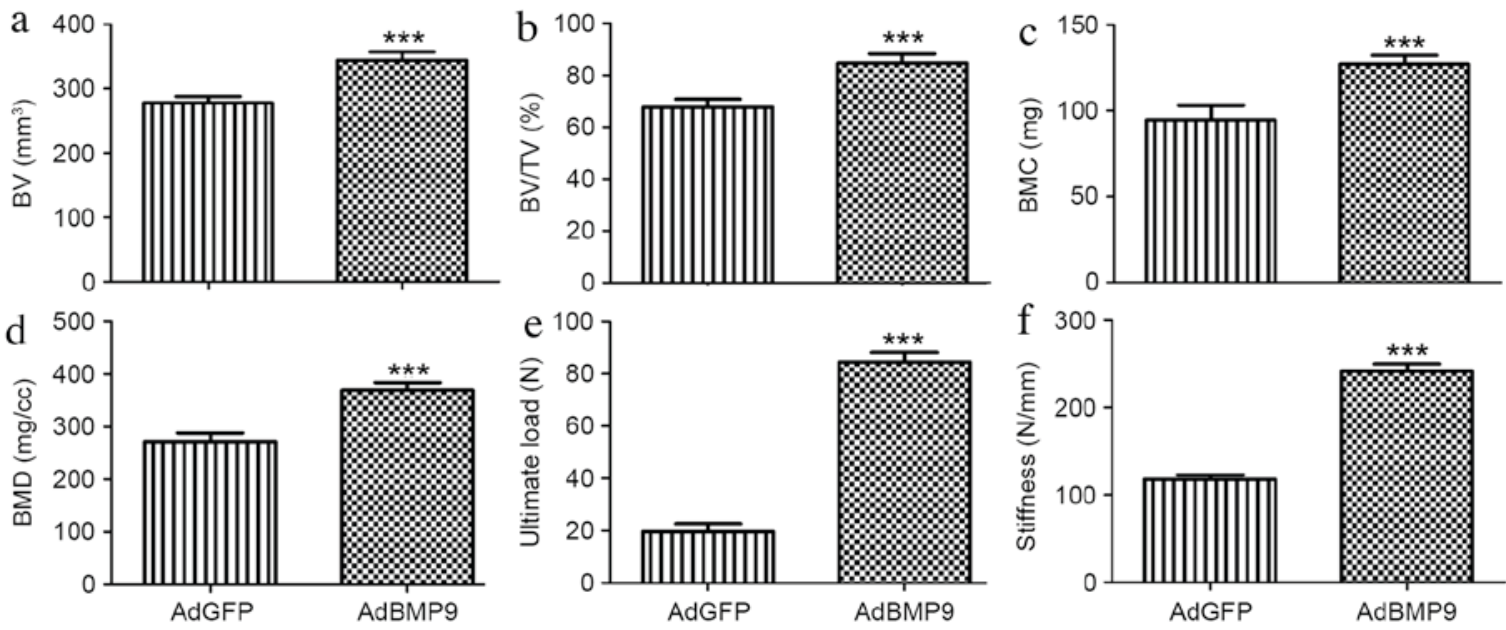

Figure 3. Morphological and micro-CT analysis of fracture healing 4 weeks after surgery. (A) The morphology of the fractured femurs was determined by (a) radiography and (b) transverse, sagittal and coronal micro-CT 3D reconstructions of the fracture. (B) Quantitative results of micro-CT analysis presented as (a) BV, (b) BV/TV, (c) BMC and (d) BMD, and biomechanical results demonstrating (e) ultimate load force and (f) stiffness. Data are presented as the mean \pm standard error $(n=5) .{ }^{* * * *} \mathrm{P}<0.001$ vs. AdGFP control group. $\mathrm{CT}$, computed tomography; $\mathrm{B}$, bone; $\mathrm{V}$, volume; $\mathrm{T}$, total; BM, bone mineral; $\mathrm{C}$, content; $\mathrm{D}$, density; GFP, green fluorescent protein; Ad, adenoviral; BMP9, bone morphogenic protein 9.

These results demonstrated that AdBMP9 locally applied via gelatin sponges may improve callus formation and enhance bone-healing ability in osteoporotic rats.

MSCs are important osteogenic progenitors that are regulated by osteogenic factors, and may be used for bone tissue engineering. BMPs are a family of growth factors considered to serve a pivotal role in bone formation. A previous study demonstrated that exposure of MSCs to osteogenic BMPs resulted in increased osteoblastic differentiation, as indicated by upregulated expression levels of osteoblast-specific markers (25). 
A

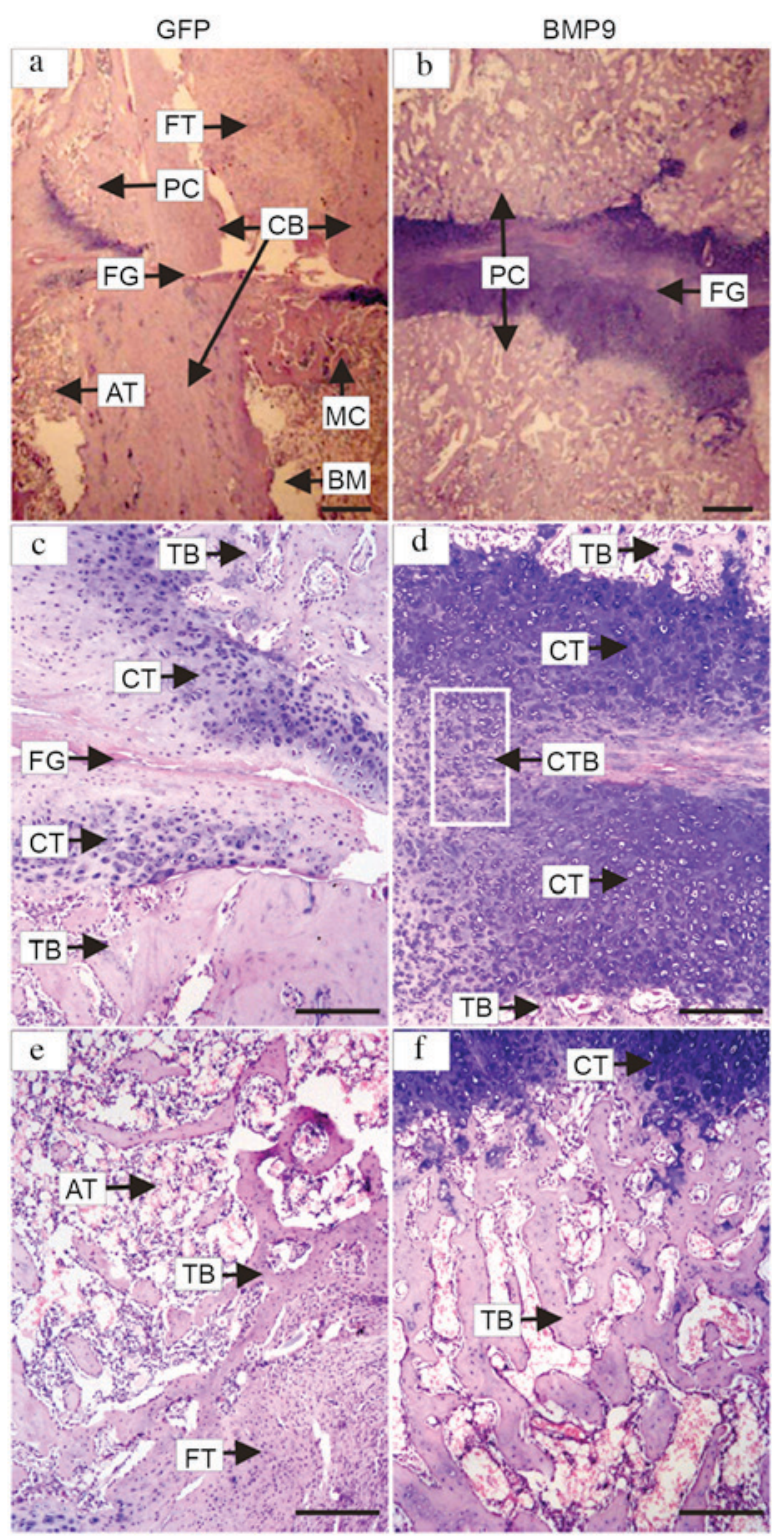

$\mathrm{B}$

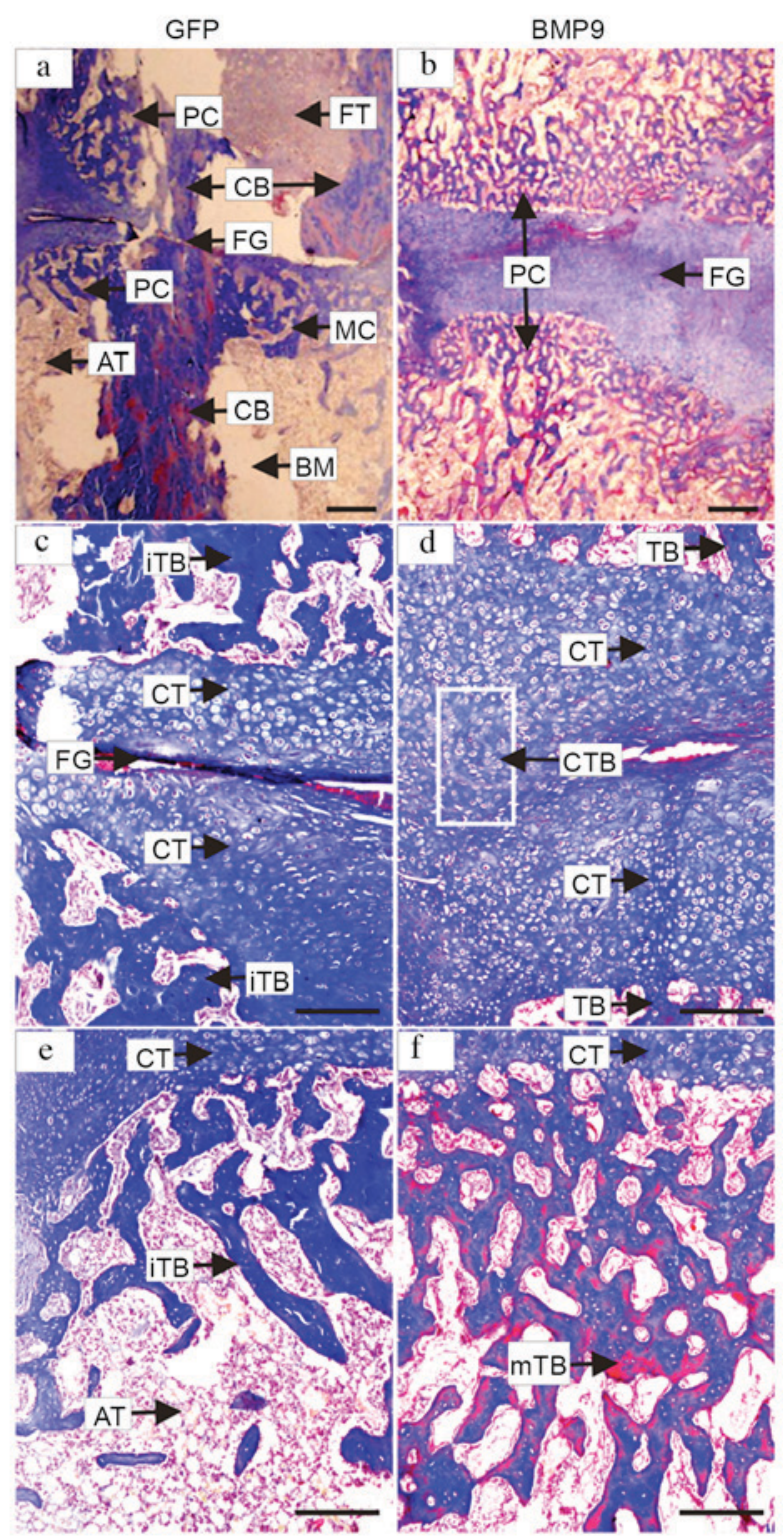

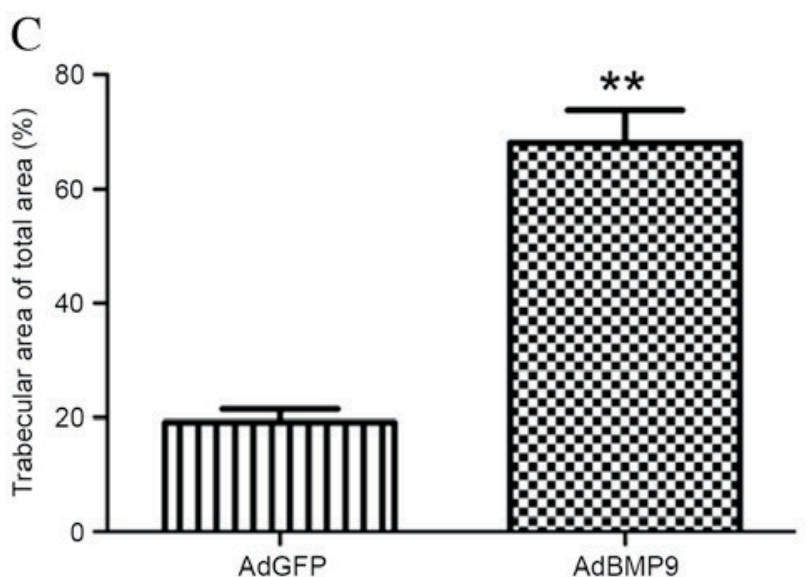

Figure 4. Histological analysis of fracture healing 4 weeks post-surgery. Longitudinal sections of the fractured femora stained with (A) hematoxylin and eosin and (B) Masson's trichrome. Representative images in (a-d) reveal the fracture gap and in (e and f) the local trabecular bone at the proximal end of fracture site. (a, c and e) Representative images from the GFP group reveal the PC, FG, AT, CB, MC, BM, less CT, fewer TB and iTB, and that the distribution of FT was prominent in the nonunion bridge area. ( $b, d$ and f) The BMP9 group demonstrated fusion of the CTB and a larger mTB around the fracture gap. (C) Quantitative analysis of trabecular area percentage of the total area. Data are expressed as the mean \pm standard error. ${ }^{* *} \mathrm{P}<0.01 \mathrm{vs}$. AdGFP control group. (a and b) Magnification, x12.5; scale bar=1000 $\mu \mathrm{m}$. (c-f) Magnification, $\mathrm{x} 40$; scale bar=250 $\mu \mathrm{m}$. PC, periosteal callus; FG, fracture gap; AT, adipose tissue; $\mathrm{CB}$, cortical bone; MC, marrow callus; $\mathrm{BM}$, bone marrow; $\mathrm{CT}$, cartilaginous tissue; $\mathrm{TB}$, trabecular bones, i, immature; m, mature; FT, fibrous tissue; CTB, cartilaginous tissue bridge; GFP, green fluorescent protein. 

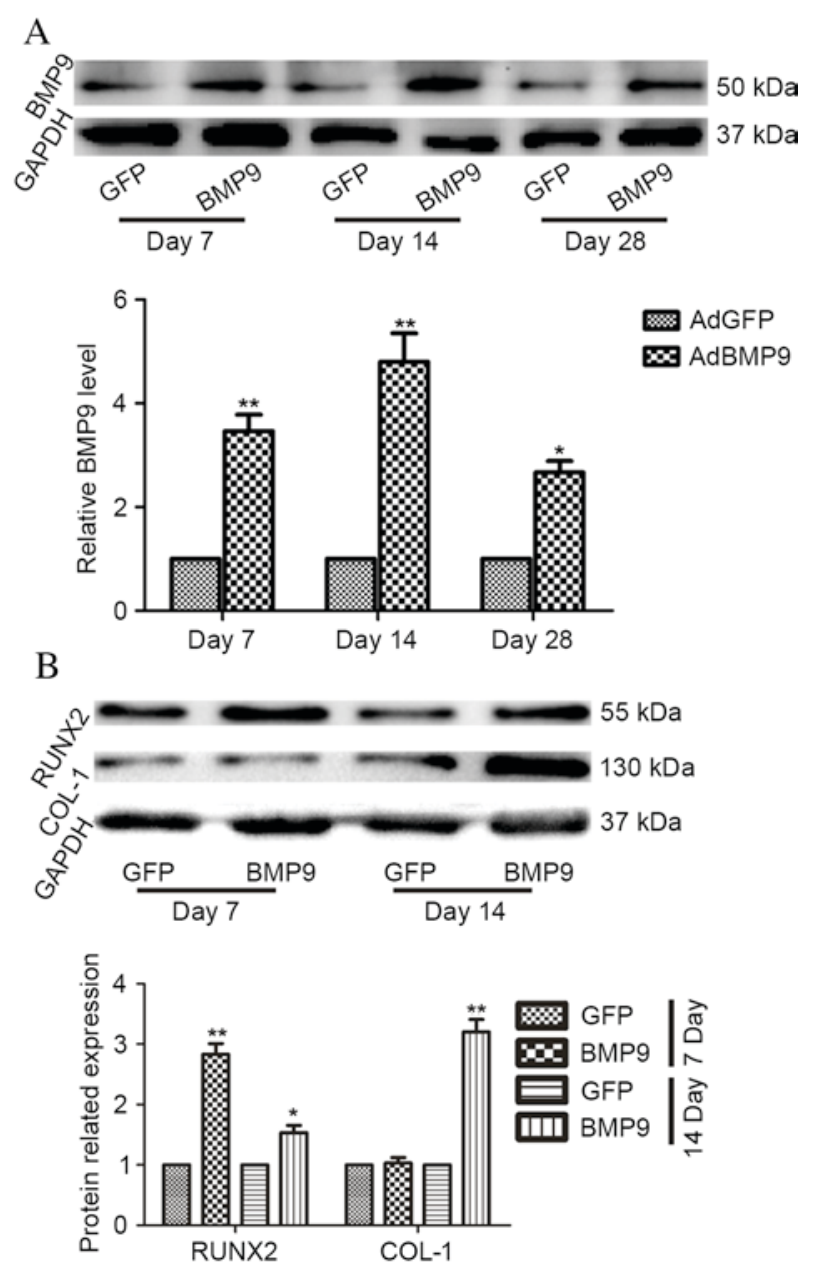

Figure 5. BMP9 improves callus formation by mediating osteoblastic differentiation in vivo. Representative bone callus western blot images and analysis of (A) BMP9 protein expression levels at days 7, 14 and 28 post-fracture and (B) RUNX2 and COL-1 protein expression levels at days 7 and 14 post-fracture. Data are expressed as the mean \pm standard error. ${ }^{*} \mathrm{P}<0.05$ and ${ }^{* *} \mathrm{P}<0.01$ vs. AdGFP control group. BMP9, bone morphogenic protein 9; RUNX2, runt-related transcription factor 2; COL-1, type 1 collagen; GFP, green fluorescent protein.

BMP9, additionally known as growth differentiation factor 2, is one of the most important bone-forming BMPs; however, its precise role in the skeletal system remains to be fully elucidated $(4,26)$. A previous study demonstrated that BMP9 may induce osteogenic differentiation (27). Additionally, a previous in vivo study revealed that injection of athymic nude mice with BMP9 or BMP9-transduced cells induced bone formation (4). Furthermore, BMP-9 has been reported to successfully induce spinal fusion and repair of nonunion bone fractures in rat models. In a study by Dumont et al (8), percutaneous paraspinal injection of AdBMP9-transduced MSCs resulted in significant ectopic bone formation and successful spinal fusion. A study by Kimelman-Bleich et al (28) indicated that in vivo electroporation of a BMP9 plasmid, in combination with recruitment of host progenitor cells, induced fracture repair in mouse models of nonunion of the radius. However, to the best of our knowledge, few studies have investigated the osteogenic effects of AdBMP9 in a rat model of osteoporotic fracture. A recent study by Shui et al (9) indicated that subcutaneous implantation of BMP9-transduced preosteoblastic cells with a COL-1 sponge scaffold demonstrated robust and mature cancellous bone masses, compared with a demineralized bone matrix carrier or direct subcutaneous injection. Therefore, the present study investigated healing in a rat osteoporotic fracture model, following the implantation of gelatin sponges containing AdBMP9. A previous study demonstrated that the process of fracture healing in rats is similar to that in other mammals; at day 21, endochondral bone formation is almost complete and bone remodeling begins (29). Scaffolds containing AdBMP7 have been revealed to maintain a stable release of BMP7 for $>21$ days in vitro, and BMP7 concentrations peaked after 14 days (30). Therefore, protein expression levels of BMP9 were investigated at various time points in AdBMP9-transfected MSCs and in rats with osteoporotic fractures implanted with sponges containing AdBMP9. The present study demonstrated that protein expression levels of BMP9 were significantly upregulated by AdBMP9, peaking at 14 days post-implantation. In addition, the effects of AdBMP9 on the microstructure, histology and biomechanics of bony callus were evaluated 28 days post-implantation. AdBMP9 in gelatin sponges was identified to increase callus formation and improve its mechanical properties during the early stages of fracture healing. Additionally, AdBMP9 was demonstrated to induce ectopic bone formation, consistent with a previous study, which indicated that BMP9 initiated the process of bone formation following percutaneous injection into the thigh musculature (31).

BMP9 has been suggested to mediate osteogenesis via signaling pathways unique from other BMPs. A previous study reported that BMP9 activated the expression of osteogenic genes, including RUNX2, and a master regulatory gene in MSC osteoblast differentiation, COL-1 (32). RUNX2 has been demonstrated to contribute to BMP9-induced ectopic bone formation (33). The findings of the present study revealed that overexpression of BMP9 upregulated RUNX2 and COL-1 expression levels in fractured bone callus. These characteristics corresponded with the histological appearance of trabecular bones in the AdBMP9 group. These results indicated that endochondral and intramembranous bone formation were promoted by BMP9 during early fracture healing.

However, the present study had various limitations. Animals were only assessed at 4 weeks post-fracture, consistent with our and others previous studies $(9,30)$; however, the fracture repair effect of BMP9 was demonstrated by the present study. In future studies, the release of BMP9 by AdBMP9 and fracture healing should be examined at additional time points. Furthermore, the cortices were not clearly bridged with bone callus in the two groups. Osteoporotic and unstable fracture factors may be primary reasons for improper healing. Nevertheless, the callus bridge gradually formed in the AdBMP9 group, as demonstrated by histology. In the control group, the fracture line was visible and fibrous tissue filled the fracture gap. Therefore, BMP9 may improve the maturation and formation of bone and cartilage tissue, and mediate callus formation and remodeling.

In conclusion, the results of the present study demonstrated that after 4 weeks, AdBMP9 in gelatin sponges directly mediated callus formation, and improved bone mass and strength in osteoporotic rats with femora fractures. However, the efficacy and safety of BMP9 administration in large animals 
and humans remains unclear and requires further investigation. Despite the limitations of the present study, the effects of BMP9 implicate it as a potential novel therapeutic target for fracture healing.

\section{Acknowledgements}

The present study was supported by the National Natural Science Foundation of China (grant nos. 81272005 to Z.-L.D and 31000434 to L.C.) and the Nature Science Foundation of Chongqing (grant no. 2013jjB10021 to Z.-L.D.).

\section{References}

1. Johnell $\mathrm{O}$ and Kanis $\mathrm{J}$ : Epidemiology of osteoporotic fractures. Osteoporos Int 16 (Suppl 2): S3-S7, 2005.

2. Song T, Wang W, Xu J, Zhao D, Dong Q, Li L, Yang X, Duan X, Liang Y, Xiao Y, et al: Fibroblast growth factor 2 inhibits bone morphogenetic protein 9-induced osteogenic differentiation of mesenchymal stem cells by repressing Smads signaling and subsequently reducing Smads dependent up-regulation of ALK1 and ALK2. Int J Biochem Cell Biol 45: 1639-1646, 2013.

3. Lamplot JD, Qin J, Nan G, Wang J, Liu X, Yin L, Tomal J, Li R, Shui W, Zhang H, et al: BMP9 signaling in stem cell differentiation and osteogenesis. Am J Stem Cells 2: 1-21, 2013.

4. Kang Q, Sun MH, Cheng H, Peng Y, Montag AG, Deyrup AT, Jiang W, Luu HH, Luo J, Szatkowski JP, et al: Characterization of the distinct orthotopic bone-forming activity of 14 BMPs using recombinant adenovirus-mediated gene delivery. Gene Ther 11: 1312-1320, 2004.

5. Hu N, Jiang D, Huang E, Liu X, Li R, Liang X, Kim SH, Chen X, Gao JL, Zhang H, et al: BMP9-regulated angiogenic signaling plays an important role in the osteogenic differentiation of mesenchymal progenitor cells. J Cell Sci 126: 532-541, 2013.

6. Wang J, Zhang H, Zhang W, Huang E, Wang N, Wu N, Wen S, Chen X, Liao Z, Deng F, et al: Bone morphogenetic protein-9 effectively induces osteo/odontoblastic differentiation of the reversibly immortalized stem cells of dental apical papilla. Stem Cells Dev 23: 1405-1416, 2014.

7. Helm GA, Alden TD, Beres EJ, Hudson SB, Das S, Engh JA, Pittman DD, Kerns KM and Kallmes DF: Use of bone morphogenetic protein-9 gene therapy to induce spinal arthrodesis in the rodent. J Neurosurg 92 (Suppl 2): S191-S196, 2000.

8. Dumont RJ, Dayoub H, Li JZ, Dumont AS, Kallmes DF, Hankins GR and Helm GA: Ex vivo bone morphogenetic protein-9 gene therapy using human mesenchymal stem cells induces spinal fusion in rodents. Neurosurgery 51: 1239-1245, 2002.

9. Shui W, Zhang W, Yin L, Nan G, Liao Z, Zhang H, Wang N, Wu N, Chen X, Wen S, et al: Characterization of scaffold carriers for BMP9-transduced osteoblastic progenitor cells in bone regeneration. J Biomed Mater Res A 102: 3429-3438, 2014.

10. Kuroda S, Sumner DR and Virdi AS: Effects of TGF- $\beta 1$ and VEGF-A transgenes on the osteogenic potential of bone marrow stromal cells in vitro and in vivo. J Tissue Eng 3 : 2041731412459745, 2012

11. Luo J, Deng ZL, Luo X, Tang N, Song WX, Chen J, Sharff KA, Luu HH, Haydon RC and Kinzler KW: A protocol for rapid generation of recombinant adenoviruses using the AdEasy system. Nat Protoc 2: 1236-1247, 2007.

12. Sosa I, Cvijanovic O, Celic T, Cuculic D, Crncevic-Orlic Z, Vukelic L, Zoricic Cvek S, Dudaric L, Bosnar A and Bobinac D: Hepatoregenerative role of bone morphogenetic protein-9. Med Sci Monit 17: HY33-HY35, 2011.

13. Tang N, Song WX, Luo J, Luo X, Chen J, Sharff KA, Bi Y, He BC, Huang JY, Zhu GH, et al: BMP-9-induced osteogenic differentiation of mesenchymal progenitors requires functional canonical Wnt/beta-catenin signalling. J Cell Mol Med 13: 2448-2464, 2009.

14. Luo J, Tang M, Huang J, He BC, Gao JL, Chen L, Zuo GW, Zhang W, Luo Q, Shi Q, et al: TGFbeta/BMP type I receptors ALK1 and ALK2 are essential for BMP9-induced osteogenic signaling in mesenchymal stem cells. J Biol Chem 285: 29588-29598, 2010.
15. Chen L, Jiang W, Huang J, He BC, Zuo GW, Zhang W, Luo Q, Shi Q, Zhang BQ, Wagner ER, et al: Insulin-like growth factor 2 (IGF-2) potentiates BMP-9-induced osteogenic differentiation and bone formation. J Bone Miner Res 25: 2447-2459, 2010.

16. Li RD, Deng ZL, Hu N, Liang X, Liu B, Luo J, Chen L, Yin L, Luo X, Shui W, et al: Biphasic effects of TGF $\beta 1$ on BMP9-induced osteogenic differentiation of mesenchymal stem cells. BMB Rep 45: 509-514, 2012.

17. Huang J, Yuan SX, Wang DX, Wu QX, Wang X, Pi CJ, Zou X, Chen L, Ying LJ, Wu K, et al: The role of COX-2 in mediating the effect of PTEN on BMP9 induced osteogenic differentiation in mouse embryonic fibroblasts. Biomaterials 35: 9649-9659, 2014.

18. Shen J, Leslie WD, Nielson CM, Majumdar SR, Morin SN and Orwoll ES: Associations of body mass index with incident fractures and hip structural parameters in a large canadian cohort. J Clin Endocrinol Metab 101: 476-484, 2016.

19. Wu SF and Du XJ: Body mass index may positively correlate with bone mineral density of lumbar vertebra and femoral neck in postmenopausal females. Med Sci Monit 22: 145-151, 2016.

20. Dostal AM, Arikawa A, Espejo L and Kurzer MS: Long-term supplementation of green tea extract does not modify adiposity or bone mineral density in a randomized trial of overweight and obese postmenopausal women. J Nutr 146: 256-264, 2016.

21. Zheng Y, Wang C, Zhang H, Shao C, Gao LH, Li SS, Yu WJ, $\mathrm{He} J W, \mathrm{Fu} W Z$, Hu YQ, et al: Polymorphisms in Wnt signaling pathway genes are associated with peak bone mineral density, lean mass, and fat mass in Chinese male nuclear families. Osteoporos Int 27: 1805-1815, 2016.

22. Poiana C, Carsote M, Radoi V, Mihai A and Capatina C: Prevalent osteoporotic fractures in 622 obese and non-obese menopausal women. J Med Life 8: 462-466, 2015.

23. Li YF, Zhou CC, Li JH, Luo E, Zhu SS, Feng G and Hu J: The effects of combined human parathyroid hormone (1-34) and zoledronic acid treatment on fracture healing in osteoporotic rats. Osteoporos Int 23: 1463-1474, 2012.

24. Zhao X, Wu ZX, Zhang Y, Gao MX, Yan YB, Cao PC, Zang Y and Lei W: Locally administrated perindopril improves healing in an ovariectomized rat tibial osteotomy model. PLoS One 7: e33228, 2012

25. Luu HH, Song WX, Luo X, Manning D, Luo J, Deng ZL, Sharff KA, Montag AG, Haydon RC and He TC: Distinct roles of bone morphogenetic proteins in osteogenic differentiation of mesenchymal stem cells. J Orthop Res 25: 665-677, 2007.

26. Cheng H, Jiang W, Phillips FM, Haydon RC, Peng Y, Zhou L, Luu HH, An N, Breyer B, Vanichakarn P, et al: Osteogenic activity of the fourteen types of human bone morphogenetic proteins (BMPs). J Bone Joint Surg Am 85-A: 1544-1552, 2003.

27. Luther G, Wagner ER, Zhu G, Kang Q, Luo Q, Lamplot J, Bi Y, Luo X, Luo J, Teven C, et al: BMP-9 induced osteogenic differentiation of mesenchymal stem cells: Molecular mechanism and therapeutic potential. Curr Gene Ther 11: 229-240, 2011.

28. Kimelman-Bleich N, Pelled G, Zilberman Y, Kallai I, Mizrahi O, Tawackoli W, Gazit Z and Gazit D: Targeted gene-and-host progenitor cell therapy for nonunion bone fracture repair. Mol Ther 19: 53-59, 2011.

29. Schmidmaier G, Wildemann B, Melis B, Krummrey G, Einhorn TA, Haas NP and Raschke M: Development and characterization of a standard closed tibial fracture model in the rat. Eur J Trauma 30: 35-42, 2004.

30. Zhang Y, Wu C, Luo T, Li S, Cheng X and Miron RJ: Synthesis and inflammatory response of a novel silk fibroin scaffold containing BMP7 adenovirus for bone regeneration. Bone 51: 704-713, 2012.

31. Li JZ, Hankins GR, Kao C, Li H, Kammauff J and Helm GA: Osteogenesis in rats induced by a novel recombinant helper-dependent bone morphogenetic protein-9 (BMP-9) adenovirus. J Gene Med 5: 748-756, 2003.

32. Bergeron E, Senta H, Mailloux A, Park H, Lord E and Faucheux N: Murine preosteoblast differentiation induced by a peptide derived from bone morphogenetic proteins-9. Tissue Eng Part A 15: 3341-3349, 2009.

33. Kang Q, Song WX, Luo Q, Tang N, Luo J, Luo X, Chen J, Bi Y, He BC, Park JK, et al: A comprehensive analysis of the dual roles of BMPs in regulating adipogenic and osteogenic differentiation of mesenchymal progenitor cells. Stem Cells Dev 18: 545-559, 2008. 